\title{
34 Rapid Prototyping in Orthopaedics: Principles and Applications
}

Jamaluddin Abdullah and Ahmad Yusoff Hassan

\subsection{Introduction}

Rapid prototyping (RP) is primarily concerned with automated fabrication of tangible model or prototype from computerised data or any computer aided design (CAD) system for visualisation, testing and verification. Physical model is a better visualisation tool as compared to two-dimensional (2D) and three-dimensional (3D) computer generated drawings or scanned images on the computer screen. As opposed to software methods which give illusion of 3D volumes on a 2D screen that can cause problems through view angle, depth and transparency, in physical modelling the user can touch and comprehend the object, manipulate it and have a feeling about its weight, size and texture (Sanghera et al., 2001). A picture can tell a thousand words, while a model is worth a thousand pictures. This becomes even more critical when the object to be analysed comprises of many complex features, such as holes, tiny lines, irregular shapes and contours and a very important decision has to be made. Biological structures and human anatomy are examples of complex objects.

In the beginning of its inception, rapid prototyping was mainly used for product development in the manufacturing industry. It was used to 


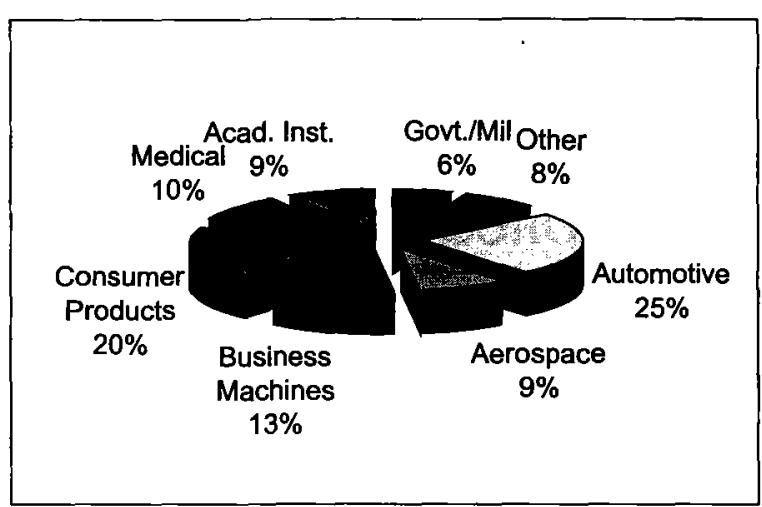

Fig. 1: User of rapid prototyping (Courtesy of Terry Wohler and Associates, 1999).

speed up design process for household products, toys, tools and mechanical components. Rapid prototyping is widely used in many other science and engineering applications, with the medical sector representing 10\% in 1999 (Fig. 1) of users (Wohler, 1999) and the usage is increasing (Wimpeny, 2001a). CAD designed objects are composed of standard features and curves. No matter how complicated CAD objects are, they are defined by numbers of basic geometric shape such as planes, cylinders, cones, etc. However, biological artifacts or the human anatomy are free-form surfaces with multiple curves and it is also unique from one person to another. These objects are difficult or impossible to replicate using traditional machining method such as milling, turning, etc. Figure 2 shows examples of simple and complicated CAD designed objects. Figure 3 shows complex medical models. With the rapid progress and advancement in computing power and related technology, rapid prototyping is able to produce complex, non-standard features from scanned data such as magnetic resonance imaging (MRI) and computer tomography (CT) data. SLA models are reported as the most widely used in the medical sector as they are ideally suited to surgical applications (Wimpeny, 2001b).

\subsubsection{Rapid Prototyping Principle}

Rapid prototyping works on the basis of adding or removing layers of material to form the desired shape. The majority of commercial rapid prototyping

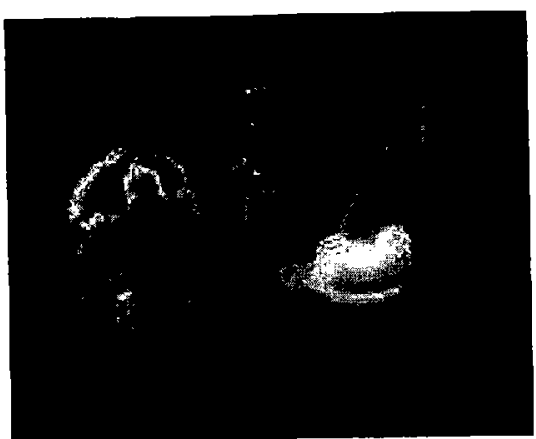

(a)

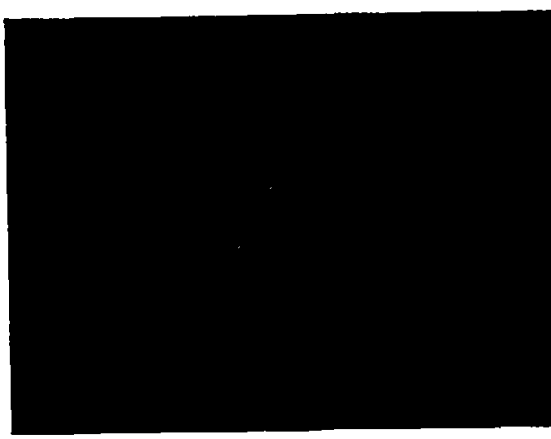

(b)
Fig. 2: Designed objects on CAD system produced using RP. (a) Complicated object. (b) Simple geometric object.

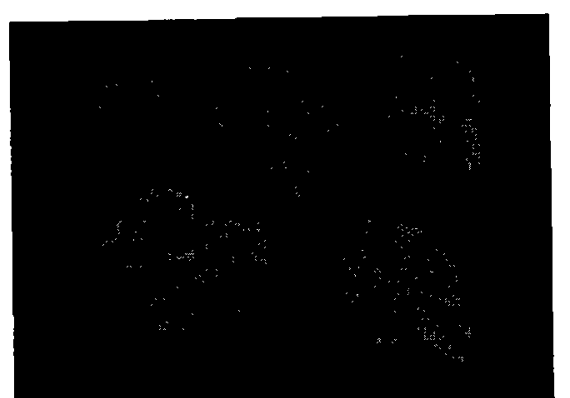

(a)

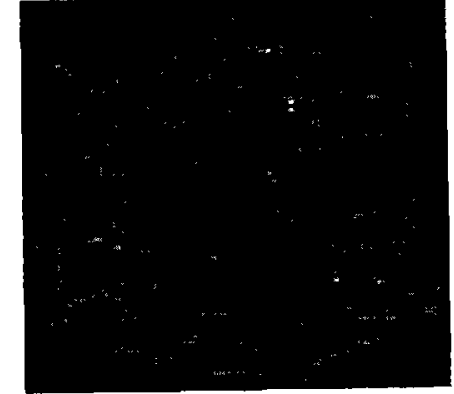

(b)

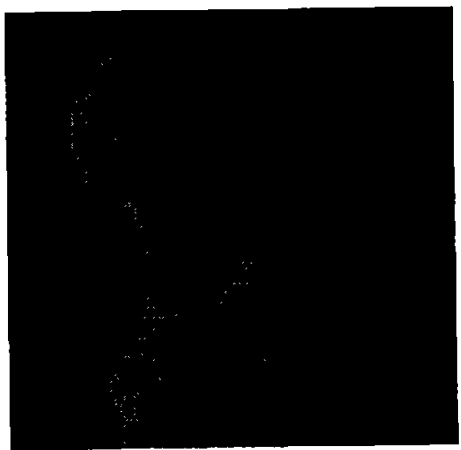

(c)

Fig. 3: (a) Cranial models produced on RP (FDM). (b) Osteoporotic bone model produced on RP (SLA). (c) Hip bone model. These are complex objects characterised by surfaces with multiole curves. 
system build object by adding one layer after another. For simplicity, it can be visualised as stacking slices of bread until a complete three-dimensional bread loaf is achieved. This process is illustrated in Fig. 4. Rapid prototyping is a highly automated layer manufacturing process.

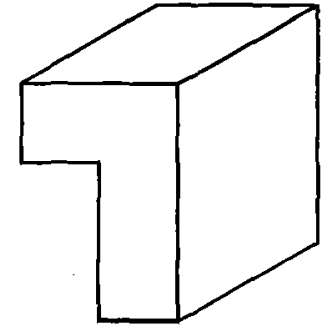

(a)

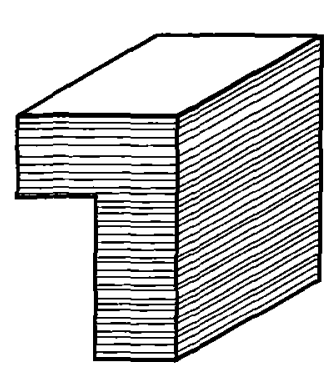

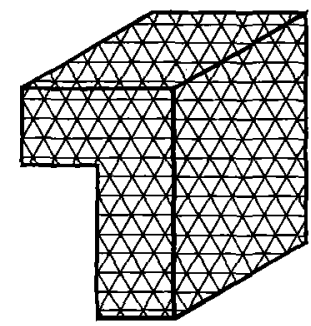

(b)

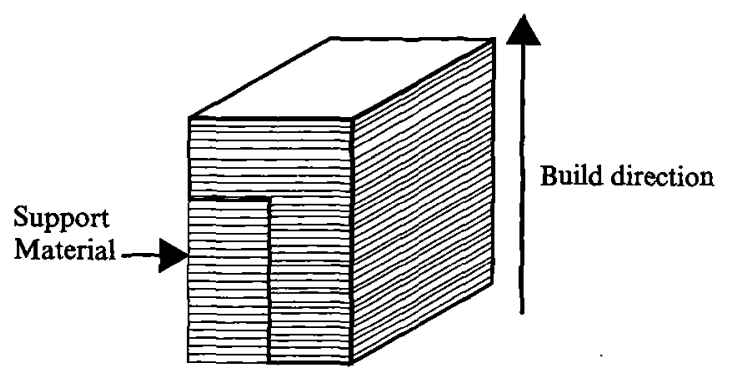

(d)

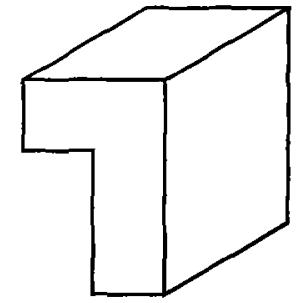

Fig. 4: Rapid prototyping process: (a) CAD solid model (b) STL model (c) Sliced models on computer (d) 3D object with support (e) Completed 3D object with support removed.
The object is designed in any solid modelling software (CAD) and the data is converted into a standard format widely known as standard triangularisation language (STL) which is understandable by the rapid prototyping machine. Rapid prototyping software receives data in this format and creates a complete set of instructions for fabrication on rapid prototyping machine such as tool path, layer thickness, processing speed, etc. Rapid prototyping machine then manufactures the object using layer manufacturing method. Upon completion of a three-dimensional model, it is subjected to post-processing treatment for removing support material that was used to support overhang features during fabrication. Depending on the rapid prototyping system and user requirements, the model may require finishing work such as cleaning, painting or curing in oven. A variety of model materials are available depending on the RP system used as shown in Table 1. A range of machine capabilities, fabrication techniques and estimated capital cost for a few machines is also shown in Table 1.

There are many rapid prototyping systems that can be used for physical modelling. These are a few examples of rapid prototyping with different

Table 1: Major commercial rapid prototyping systems.

\begin{tabular}{|c|c|c|c|c|c|}
\hline $\begin{array}{c}\text { Commercial } \\
\text { System }\end{array}$ & $S L A$ & $F D M$ & $S L S$ & LOM & $3 D P$ \\
\hline Model Material & $\begin{array}{l}\text { Liquid } \\
\text { photopolymer }\end{array}$ & $\begin{array}{l}\text { ABS, } \\
\text { Wax, Teflon } \\
\text { Filament }\end{array}$ & Powder & $\begin{array}{l}\text { Paper, } \\
\text { Plastic, } \\
\text { Metal } \\
\text { Sheets }\end{array}$ & $\begin{array}{l}\text { Gypsum } \\
\text { powder, Corn } \\
\text { starch }\end{array}$ \\
\hline $\begin{array}{l}\text { Processing } \\
\text { Speed }\end{array}$ & Medium & Low & Medium & High & High \\
\hline $\begin{array}{l}\text { Maximum part } \\
\text { size }(\mathrm{mm})\end{array}$ & $\begin{array}{l}508 \times 508 \times \\
610\end{array}$ & $\begin{array}{l}610 \times 508 \times \\
610\end{array}$ & $\begin{array}{l}381 \times 330 \times \\
457\end{array}$ & $\begin{array}{l}812 \times 559 x \\
508\end{array}$ & $\begin{array}{l}508 \times 609 x \\
406\end{array}$ \\
\hline Accuracy (mm) & $0.05-0.1$ & $0.1-0.3$ & $0.1-0.2$ & 0.25 & $0.2-0.3$ \\
\hline $\begin{array}{l}\text { System Price } \\
\text { (USD) } \times 1000\end{array}$ & $75-800$ & $30-300$ & 300 & $120-240$ & $30-70$ \\
\hline $\begin{array}{l}\text { Fabrication } \\
\text { technique }\end{array}$ & $\begin{array}{l}\text { Laser curing } \\
\text { of liquid } \\
\text { photopolymer }\end{array}$ & $\begin{array}{l}\text { Fused } \\
\text { deposition of } \\
\text { molten } \\
\text { polymer }\end{array}$ & $\begin{array}{l}\text { Selective } \\
\text { laser tracing } \\
\text { of powder } \\
\text { layer }\end{array}$ & $\begin{array}{l}\text { Laser cutting } \\
\text { and stacking } \\
\text { of glued } \\
\text { sheets }\end{array}$ & $\begin{array}{l}\text { Adhesive/glue } \\
\text { Bonding of } \\
\text { powder by } \\
\text { inkjet }\end{array}$ \\
\hline
\end{tabular}


method of forming three-dimensional objects. However, no single rapid prototyping alone is dominant in medical applications. As model material varies and consequently their strength and properties also vary from one system to another, users will find that one system alone is not always the best choice in every condition. For example, fused deposition modelling (FDM) machine is frequently used for producing plastic model which is used as casting pattern to manufacture custom implant while stereolithography apparatus (SLA), a machine that uses photo-curable polymer material, is used to produce a model that is transparent with colour coded on selected diagnostic area and can effectively reveal internal features of bones or organs. The SLA model is also easy to sterilise, allowing the model to be brought into an operating room without much hassle. In orthopaedics, producing a model of actual size and form exceeds the need for high model accuracy, and therefore, a rapid prototyping system with bigger work envelope and higher processing speed is more favourable. However, most of the time, the intended usage of a rapid prototyped model greatly influence which rapid prototyping system is adopted.

A brief description of each rapid prototyping technique is given in the following sections.

\subsubsection{Stereolithography Apparatus (SLA)}

SLA machine accept part as an .stl file and slices the file into thin layers, typically from $0.10 \mathrm{~mm}-0.15 \mathrm{~mm}$. The part is built in a vat of resin by selectively curing a layer of photocurable resin that sits above a Z-stage elevator. Laser is used to scan the surface of resin and cure the resin along laser traces. When one layer is completed, the platform drops lower into the vat of resin. Fresh resin floods over the cured layer and the next layer build up continues. When all layers are completed, the part is cleaned and post-cured.

\subsubsection{Fused Deposition Modelling (FDM)}

FDM machine builds part by extruding a semi-molten filament through a heated nozzle onto a platform. When one layer is complete, the platform moves down by one layer thickness and the process of extruding another

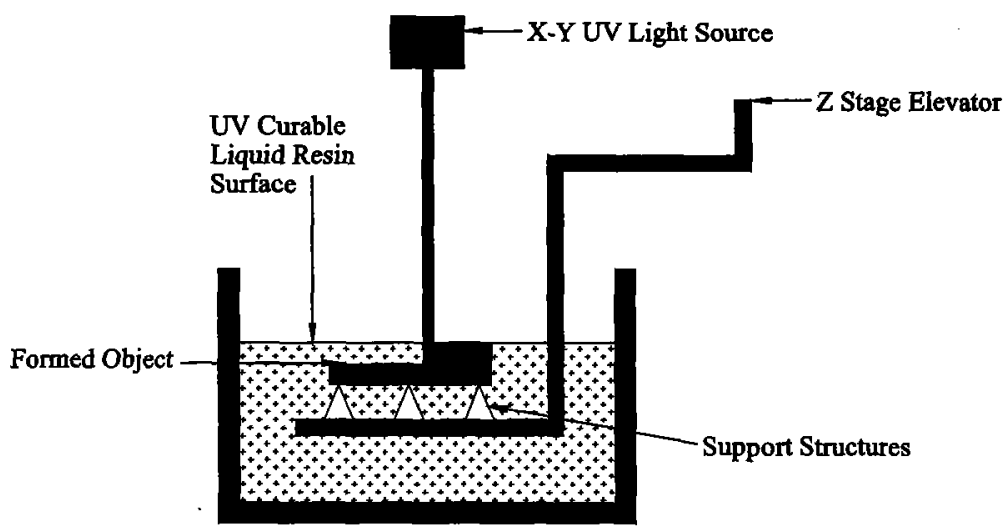

Fig. 5: SLA process.

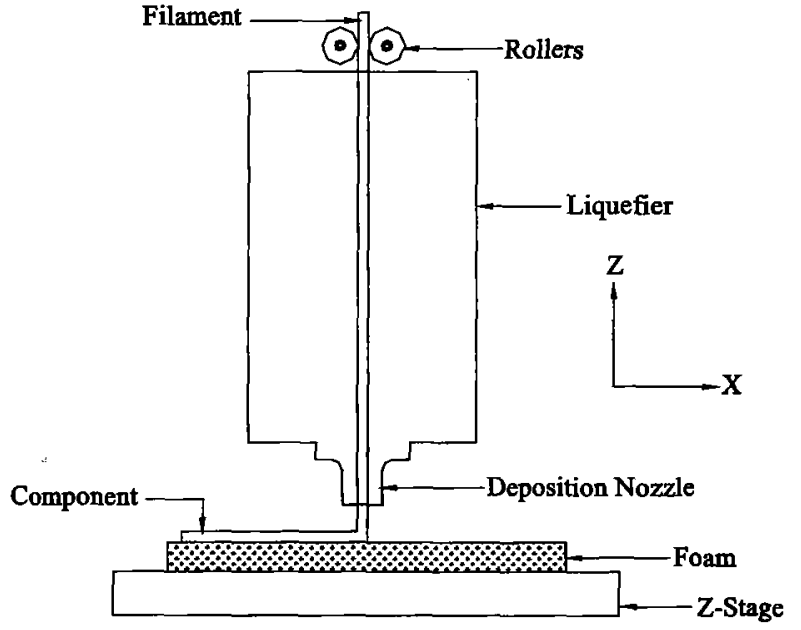

Fig. 6: FDM process.

layer continues. After completion of all layers, the part is removed from the platform and support material can be peeled off or it can be removed by ultrasonic vibration and solvent in an ultrasonic tank. This is desirable for parts with internal cavities which are not accessible by hands. 


\subsubsection{Selective Laser Sintering (SLS)}

SLS works by selectively fusing a layer of powder material on a powder bed enclosed within a build chamber. A powder supply roller supplies layer of powder onto the work area, then carbon dioxide laser scans and fuses the layer. The fused layer is lowered into a part build chamber. The process is repeated until a complete part is formed. Once completed, the part is removed from the build chamber and the loose powder is removed and reused.

\subsubsection{Laminated Object Manufacturing (LOM)}

In LOM, sheets with single-sided heat activated glue is supplied from a sheet supply roll onto the platform. Laser is used to cut cross-sectional outline of the layer. A new layer is bonded to the previously cut layer and a new cross-section is created and cut. Once all layers have been laminated and cut, excess material is removed to expose the finished model.

\subsubsection{Three-Dimensional Printing (3DP)}

The three-dimensional printing system receives input CAD file in either STL, DXF and HPGL format. Sliced information is then created as in other

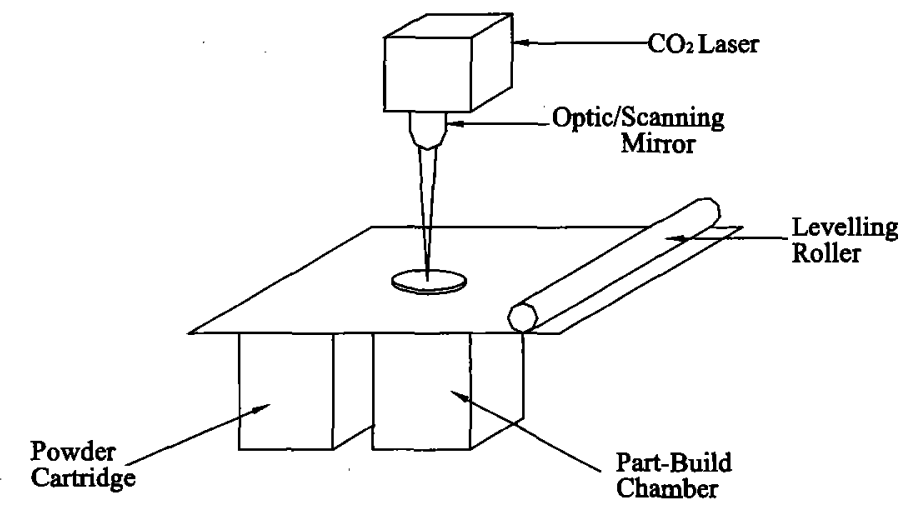

Fig. 7: SLS process.

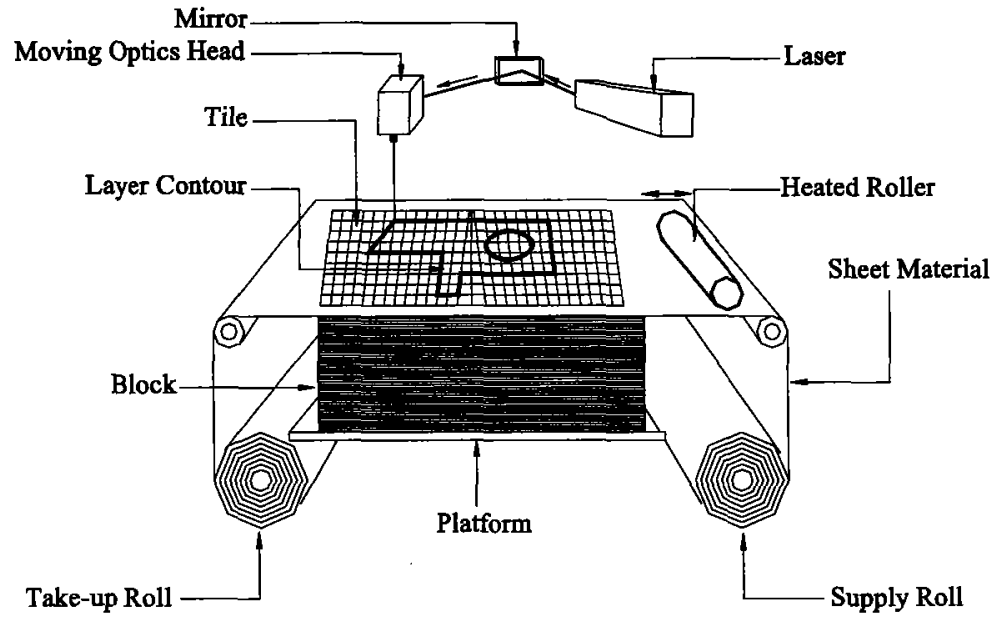

Fig. 8: LOM process.

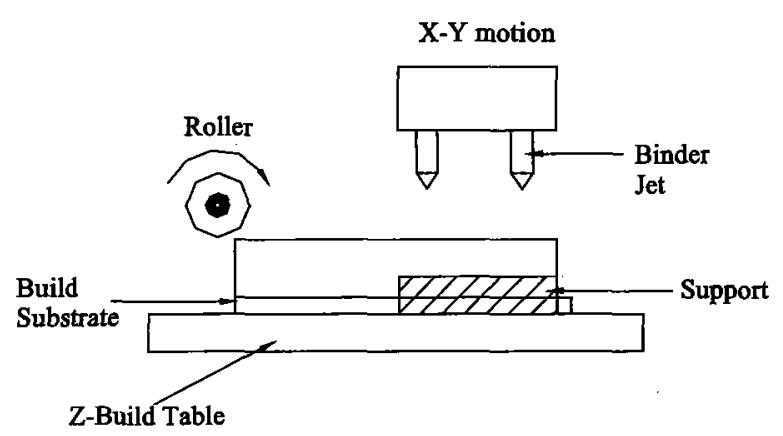

Fig. 9: 3DP process.

systems. Powder is supplied on a platform and a roller spreads the powder layer evenly. Printing head as in inkjet printer, releases jets of binder to bond powder material. The platform is lowered one layer thickness and the next layer is built until the part is completed. One advantage of 3DP is that, no support structure is needed. Support is naturally provided by unused powder around the bonded powder particle. Upon completion, the part is removed and unused powder is removed by shaking the part gently. 


\subsection{Applications of Rapid Prototyping in Orthopaedics}

Production of prototypes for medical modelling (orthopaedics) in general can be classified into two broad categories based on manufacturing process route and type of data available, i.e. designed data and scanned/digitised data. Designed data is data that is created according to a person's idea on computer aided design (CAD) system. For this type of data, the designer has total control to modify, adjust and manipulate his design ideas to serve the functional purpose of his design. Producing models with this type of data is very straightforward and no further data treatment is required. CAD solid model can be directly converted to STL format for use in subsequent rapid prototyping process.

Scanner or digitiser is normally used to capture structures that exists in physical form, either dead or living things, and using surface modeller software, three-dimensional CAD representation is created. For this type of data, the user has limited capability to modify and manipulate the geometry and further processing is required before they can be readily used by rapid prototyping system. For example, further data treatment is needed for scanned data from computed tomography (CT) and magnetic resonance imaging (MRI) scanners which capture soft and hard tissue information based on density threshold value. The undesired soft tissue data is removed before it is sent to rapid prototyping machine for fabrication. Segregating soft tissue data and leaving only hard tissue (i.e. bone) structure can be carried out by applying certain range of density threshold value. This procedure can be a daunting task for complex structure and one has to repeat the procedure many times until satisfactory result is achieved. There are a number of commercial softwares such as MIMICS, and Go-build which translate this data to the format required by RP systems. In reverse engineering method, point cloud data for an existing object is captured using coordinate measuring machine or laser digital surface scanner and using surface modeller, this raw data is processed to form three-dimensional model of the object in CAD system. The roadmap from data capture to orthopaedics modelling is illustrated in Fig. 10.

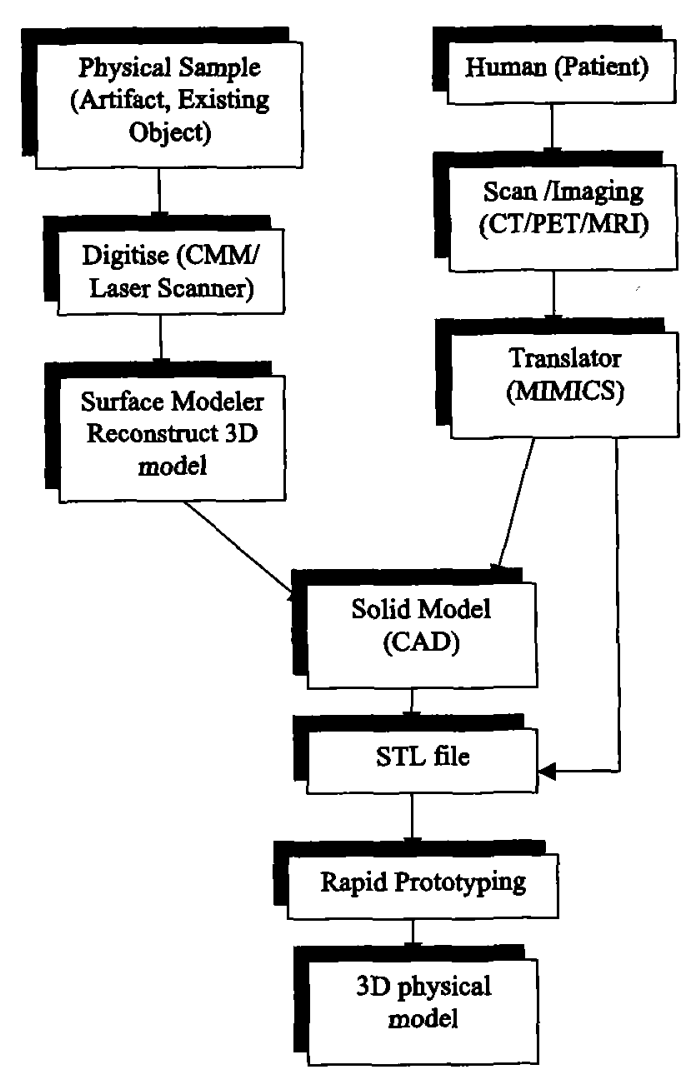

Fig. 10: Roadmap for orthopaedics modelling.

\subsubsection{Development of Medical Devices and Instrumentation}

One of the most obvious applications of rapid prototyping in the medical sector is as a means to develop and manufacture medical devices and instrumentation. It is applied in any field where time reduction is needed for development, while simultaneously providing users with functional performance feedback. This becomes very critical in the medical sector, where human lives, to some extend depend on the quality and ease of use of 
numerous medical products. Instruments like retractors, scalpels, surgical fasteners and many other devices have been designed using rapid prototyping technology.

It is found that by applying RP in development of surgical tool, cost and time for design iteration is reduced, the part count for final assembly is minimised and a more elegant design is achieved. From a case study that compares the traditional casting and machining method with RP technology, a better final design is achieved (lighter, cheaper, more ergonomic) by utilising RP techniques for some stages of the development process (Jamieson et al., 1995). This is particularly vital in the surgeon acceptance stage which is necessary prior to product manufacturing. New innovative surgical equipments are being developed for use in orthopaedics and reconstructive surgery.

\subsubsection{Visualisation and Training, Diagnostic and Surgery Planning}

In orthopaedics, perhaps the most widely used applications of rapid prototyping until recent time is as a means to quickly model the human bones for visualisation and training, enhanced diagnostic, surgery planning of complex procedures and as reference template in operating theater. With better visualisation on the diagnosed region, proper training, planning and rehearsal can be carried out before surgery. Some surgeons prefer a physical model for visualisation and therefore can make better judgement on further treatment plans (D'Urso et al., 2000; Webb, 2000; Petzold et al., 1999; Winder et al., 1999). These practices can lead to improved surgery results, less blood loss and faster recovery for the patient.

\subsubsection{Custom Prosthesis and Implant}

Development of prosthesis and implant is also greatly improved by rapid prototyping. Previously, hip replacement and other surgeries were carried out using standard sized replacement parts selected from a range provided by manufactures based on anthropomorphic data. This works well for some types of procedures, but not all. There are patients outside the standard range, between sizes, or with special requirements caused by disease or genetics. With rapid prototyping, it is now possible to manufacture a custom prosthesis that precisely fits a patient at reasonable cost.

One interesting area where rapid prototyping can play a significant role in implant is in the area of advanced material. Developments are taking place to develop new bio-compatible material that can be used for implant (for example as bridge between fracture bones or as direct bone replacement). Materials such as hydroxyapatite (HA) (Furukawa et al., 2000) and sea corals are being tested for possible use in humans.

\subsubsection{Limitations of Current Technology}

Rapid prototyping is still an expensive technology for use by the majority of patients, particularly for customised item which are produced in a very small quantity. High capital investment, material costs and maintenance are major contributing factors to the higher cost per model. Machine run time also contribute to total cost calculation. Therefore, the decision to use rapid prototyping is based on selective cases only. Sometimes, it is more cost effective to resort to the existing practice and using rapid prototyping is not necessary. Furthermore, at the current stage of the technology, rapid prototyping cannot be readily employed in an emergency situation where major treatment decision must be made within hours. The time needed for rapid prototyping machine to produce a life size model of the human body is still relatively slow. For a complex model and fine surface quality, longer fabrication time is needed.

The material of choice is still limited for most rapid prototyping system. Most use polymer/resin of some forms (ABS, epoxy, photo-curable resin) while others use organic material like starch powder. A few use wood, ceramic or plastic. Some of the materials exhibit adequate strength but are not necessarily suitable for direct use in the human body. There are some newly developed materials such as hydroxyapatite (HA) and sea corals for use in medicine that have been tested bio-compatible and maybe can be used safely in humans, but no available commercial technology can fabricate these materials directly as in rapid prototyping process into the form and strength required. Automated layer manufacturing method such as rapid 
prototyping must be able to produce custom implant from bio-compatible material. Likewise, this bio-compatible material may also be modified to suit the processing requirements of any rapid prototyping system.

\subsection{Concluding Remarks}

Using principles of rapid prototyping, CT scan data of anatomical parts, such as hip bones, can be translated into a format that can be directly used to manufacture three-dimensional medical models. This technique allows surgeons to hold accurate three-dimensional models rather than have to interpret 2D data from X-rays or CT scans which is normally not an enjoyable task. In addition to facilitating pre-operative planning, models are also used in communicating with patients so that they can make informed and accurate decisions in cases of life threatening medical treatment. It is also being increasingly used in medical-legal related cases. Another emerging area is bio-materials, where custom implant from bio-compatible materials can be directly fabricated. Research is underway to close the gap between advanced material, manufacturing method and the medical sector in order to achieve this promising breakthrough. In tissue engineering, rapid prototyping is used to produce scaffolds where life tissue is seeded and then implanted onto the patient.

\section{Acknowledgement}

The support from Universityy of Sciences Malaysia through Grant No. 0734107 is appreciated. The authors also acknowledge Mr. Najib Hussain, technical staff at the School of Mechanical Engineering for his dedicated laboratory support.

\section{References}

D'Urso PS, Earwaker WJ, Barker TM, Redmond MJ, Thompson RG, Effeney DJ and Tomlinson FH (2000) Custom cranioplasty using stereolithography and acrylic, Brit. J. Plast. Surg. 53: 200-204.
Furukawa T, Matsusue $Y$, Yasunaga T, Shikinami $Y$, Okuno $M$ and Nakamura T (2000) Biodegradable behaviour of ultra high strength of hydroxyapatite/poly (L-lactic) composite rods for internal fixation of bone fractures, Biomaterials 21: 889-898.

Jamieson R, Holmer B and Ashby A (1995) How rapid prototyping can assist in the development of new orthopaedic products - a case study, Rapid Prototyping $J$. 1: 38-41.

Petzold R, Zeilhofer H-F and Kalender WA (1999) Rapid prototyping technology in medicine - basics and applications, Comput. Med. Imag. Grap. 23: 277-284.

Sanghera B, Naique S, Papaharilaou Y and Amis A (2001) Preliminary study of rapid prototype medical models, Rapid Prototyping J. 7: 275-284.

Webb PA (2000) A review of rapid prototyping (RP) techniques in the medical and biomedical sector, J. Med. Eng. Tech. 24: 149-153.

Wimpeny D (2001) Overview of Medical Applications, Rapid Prototyping Casebook, Professional Engineering Publishing, UK, pp. 175-176.

Winder J, Cooke RS, Gray J, Fannin T and Fegan T (1999) Medical rapid prototyping and 3D CT in the manufacture of custom cranial titanium plates, J. Med. Eng. Technol. 23: 26-28.

Wohlers T (1999) Rapid Prototyping and Tooling State of the Industry, Wohlers Associates, pp. 2. 